Archives

$4 \mid 1989$

Varia

\title{
De la Grèce à Rome : transmission et évolution des techniques de changeurs-banquiers
}

Jean Andreau

\section{OpenEdition}

Journals

Édition électronique

URL : http://journals.openedition.org/ccrh/2897

DOI : $10.4000 /$ ccrh.2897

ISSN : $1760-7906$

Éditeur

Centre de recherches historiques - EHESS

Édition imprimée

Date de publication : 15 octobre 1989

ISSN : 0990-9141

Référence électronique

Jean Andreau, « De la Grèce à Rome : transmission et évolution des techniques de changeurs-

banquiers », Les Cahiers du Centre de Recherches Historiques [En ligne], 4 | 1989, mis en ligne le 13 avril 2009, consulté le 19 avril 2019. URL : http://journals.openedition.org/ccrh/2897 ; DOI : 10.4000/ ccrh.2897

Ce document a été généré automatiquement le 19 avril 2019

Article L.111-1 du Code de la propriété intellectuelle. 


\title{
De la Grèce à Rome : transmission et évolution des techniques de changeurs- banquiers
}

\author{
Jean Andreau
}

1 C'est en Grèce que sont apparus les premiers changeurs-banquiers professionnels, assurant le change et l'essai des monnaies, recevant des dépôts et prêtant l'argent de ces dépôts. Après avoir été frappée pour la première fois par les Lydiens, au cours de la seconde moitié du $\mathrm{VII}^{\mathrm{e}}$ siècle av.J.-C., la monnaie d'argent a été peu à peu frappée et utilisée dans toute la Grèce, et, au cours de la seconde moitié du vi ${ }^{\mathrm{e}}$ siècle, apparaissent les premiers changeurs (on en connaît à Byzance vers $520 \mathrm{av.J.-C.).} \mathrm{Un} \mathrm{siècle} \mathrm{plus} \mathrm{tard,} \mathrm{à}$ peu près à l'époque de Périclès, on entend parler à Athènes des premiers changeursbanquiers, appelés trapézites (les hommes de la table, du comptoir).

2 Par rapport à la péninsule grecque et à la Grande-Grèce (c'est-à-dire aux cités grecques d'Italie du Sud et de Sicile), Rome souffrait d'un net retard; elle ne frappe pas de monnaies d'argent avant le début du III $^{\mathrm{e}}$ siècle, ou, au plus tôt, avant la dernière partie du $\mathrm{IV}^{\mathrm{e}}$ siècle av. J.-C. Les premiers changeurs-banquiers, les argentarii (les hommes du métalargent), n'y apparaissent, dans les boutiques du forum, qu'entre 318 et 310 av. J.-C., un bon siècle après leur apparition à Athènes.

3 Au début, les opérations qu'ils effectuent et les techniques qu'ils utilisent sont héritées de la Grèce (peut-être par l'intermédiaire de la Grande Grèce, mais nous ne possédons, sur la vie financière privée de cette région, pratiquement aucune information). Les comédies de Plaute et de Térence, écrites à la fin $\mathrm{du}_{\mathrm{III}}^{\mathrm{e}}$ siècle et au cours de la première moitié du $\mathrm{II}^{\mathrm{e}}$ siècle av. J.-C., en font foi. Quand il y est question de change, d'essai des monnaies, de prêt, de dépôt, de changeurs et de banquiers, les allusions à la Grèce et les allusions à Rome sont sans cesse étroitement mêlées. Les allusions à la Grèce sont abondantes et circonstanciées, car ces auteurs de comédies écrivent des oeuvres de tradition grecque, des palliatae; c'est une règle du genre que la scène se passe dans une ville grecque, que les personnages portent des noms grecs et soient censés raisonner comme des Grecs. Les 
allusions à Rome ne sont guère moins fréquentes, du moins chez Plaute, car il aime à railler ceux que son public côtoie chaque jour dans les rues et sur la place centrale, par exemple les boutiquiers. On s'aperçoit clairement à la lecture que les changeurs et changeurs-banquiers grecs et romains se ressemblent comme des frères dans ces comédies et que Plaute peut se permettre de passer sans cesse des uns aux autres, en général sans expliquer à son public les us et coutumes particuliers aux Grecs (il lui arrive cependant d'expliquer certains de ces usages - par exemple dans les Bacchides, quand il est question de dépôts d'argent et d'objets précieux dans le sanctuaire d'Artémis à Ephèse).

4 Mon objectif dans ce bref exposé est de faire quelques remarques sur la transmission et l'évolution des techniques utilisées par ces changeurs et banquiers professionnels (en m'abstenant de poser l'important problème de la notion même de techniques financières : quels rapports et quelles différences établir entre de telles techniques et les techniques de production ?). Quand les techniques financières sont passées de la Grèce à Rome, se sont-elles maintenues telles quelles? Si elles ont connu par la suite une évolution ou des modifications, à quelles causes les attribuer? Jusqu'à la fin de l'Antiquité, la continuité l'a-t-elle emporté ou non dans ce domaine?

Quant à l'essai des monnaies, on constate, entre la Grèce et Rome, un exact parallélisme et une grande continuité. Pour contrôler le titre des monnaies, la meilleure méthode est celle de la pierre de touche ou pierre lydienne, connue tant en Grèce qu'à Rome et dans le monde romain.

6 La pierre lydienne est un morceau de schiste alluvial, contre lequel la monnaie à examiner est frottée; elle imprime sur la pierre des traces que l'on compare à celles laissées par un objet d'or ou d'argent au titre connu. La pierre lydienne est plus efficace pour l'or que pour l'argent. On en a découvert récemment sur plusieurs épaves de bateaux antiques, ce qui prouve que les commerçants en faisaient un usage courant. Quant aux essayeurs-changeurs de métier, ils en possédaient, mais en Grèce comme à Rome, les indices disponibles suggèrent qu'ils préféraient à cette technique un certain nombre de manipulations simples, sans l'aide d'aucun outil: l'essayeur regarde attentivement la pièce, il la jette à terre à plusieurs reprises en écoutant le son qu'elle rend; il la touche ; Epictète prétend même qu'il prête attention à son odeur. Après ces manipulations, il la pèse au moyen du trébuchet, petite balance dont l'usage traverse évidemment toute l'Antiquité pour se perpétuer jusqu'à l'époque moderne et contemporaine.

7 Cette apparente réticence devant l'emploi de la pierre lydienne témoigne de l'attitude extrêmement empirique des essayeurs professionnels, tant romains que grecs. Quant à leurs autres accessoires, il faut signaler que deux d'entre eux, attestés dans l'Empire romain, ne le sont pas dans le monde grec classique et hellénistique. L'un est un gabarit, c'est-à-dire un médaillier servant à compter plus rapidement un certain nombre de monnaies. L'autre, une planche d'échantillons, un plateau à godets, circulaires ou carrés, dans lequel l'essayeur dispose les échantillons des monnaies dont il peut avoir à faire l'essai, c'est-à-dire soit des exemplaires réels des monnaies, soit des poids monétaires ( exagia). Ces accessoires commodes se sont-ils transmis des Grecs aux Romains? Il est très possible aussi qu'ils ne soient entrés en usage qu'à l'époque romaine.

8 Les changeurs-banquiers, qui se nomment trapézites dans le monde grec, portent à Rome et dans la partie latine de l'Empire romain, plusieurs noms - argentarii, coactores argentarii, puis aussi nummularii. Ils sont en mesure de prêter l'argent qu'ils reçoivent en dépôt, et 
ces opérations de dépôt, de retrait et de prêt sont inscrites aux comptes de dépôts des clients. L'acheteur d'un bien quel qu'il soit, au marché ou ailleurs, peut, s'il le désire, s'adresser à un banquier en vue d'obtenir un prêt, afin de payer ce qu'il a acheté. Mais soulignons qu'à Rome comme en Grèce les banquiers sont loin d'être les seuls à prêter de l'argent. D'une part, les aristocrates (sénateurs, chevaliers) et le reste des notables, qui sont des propriétaires fonciers, d'autre part les commerçants prêtent souvent des sommes beaucoup plus importantes que celles dont disposent les banquiers professionnels.

9 L'argent emprunté était-il souvent destiné, d'une manière ou d'une autre, à des investissements dans la vie économique? Il semblerait plutôt que non. P. Millett, disciple de Moses Finley, prétend que cinq seulement des neuf cents prêts connus de lui en Grèce étaient des prêts productifs. Reste à savoir comment l'on définit l'adjectif " productif » et dans combien de cas la documentation disponible nous éclaire sur la destination du prêt.

Quoi qu'il en soit, une importante innovation technique se produit à Rome au cours de la seconde moitié du II ${ }^{e}$ siècle av. J.-C. Les changeurs-banquiers, les argentarii, se mettent à participer régulièrement aux ventes aux enchères et à y accorder du crédit aux acheteurs, de façon institutionnelle. L'argentarius ne se borne pas à agir en créancier à l'égard de l'acheteur; sur le plan juridique, il est partie prenante dans les rapports financiers résultant de la vente aux enchères. Quoique le contrat de vente soit conclu par l'acheteur et le vendeur, le banquier d'une part verse l'argent au vendeur (le jour de la vente, ou deux ou trois jours plus tard) et, d'autre part, se fait rembourser par l'acheteur, à l'échéance d'un prêt à intérêt qui, dans les exemples disponibles, n'excède pas un an. Si l'acheteur ne paie pas, tout indique que le risque financier (appelé periculum par les Latins) est supporté par le banquier, qui ne paraît pas avoir la possibilité de se retourner vers le vendeur. Auparavant intervenaient, dans les ventes aux enchères, outre les praecones (crieurs publics jouant grosso modo le rôle de commissaires-priseurs), des encaisseurs (coactores) qui facilitaient les paiements, mais à titre de purs et simples intermédiaires, et sans prêter eux-mêmes d'argent, du moins dans le cadre de leur métier.

11 Les banquiers travaillent dans des boutiques, mais les ventes aux enchères ont lieu soit sous les portiques du forum (à Pouzzoles, et probablement à Pompéi), soit dans des marchés, soit dans des locaux spécialement aménagés à cet effet (atria, atria auctionaria). $\mathrm{Du} \mathrm{I}^{\mathrm{er}}$ au $\mathrm{III}^{\mathrm{e}}$ siècle ap.J.-C., il est sûr que certaines d'entre elles se déroulaient dans des marchés de détail de Rome (par exemple le Macellum Magnum, ouvert en 59 ap. J.-C.) ou dans des marchés de gros, à Rome, Ostie ou Portus. Ces marchés de gros concernaient en particulier la vente en gros des vins transportés à Rome, soit par mer, soit en descendant le Tibre (forum vinarium, portus vinarius, portus vinarius superior). En dehors de la région de Rome, en Italie centrale et méridionale, certains marchés ruraux périodiques, qui, à l'époque républicaine, se tenaient tous les neuf jours (nundinae), étaient accompagnés d'enchères. Si toutes les enchères n'étaient pas en rapport avec la vie commerciale (beaucoup de notables vendaient aux enchères des objets divers, des esclaves, des meubles, des maisons et même des terres), certaines concernaient à coup sûr des denrées commercialisées; il arrivait que l'acheteur lui-même fût un commerçant et qu'ayant bénéficié d'un prêt de l'argentarius, il pût revendre avant même de s'être acquitté du prix de son achat.

Pourquoi cette transformation, par laquelle s'instaure une pratique tout à fait unique dans l'histoire, et qui se perpétue pendant plus de quatre siècles, jusqu'à la seconde 
moitié du III ${ }^{\mathrm{e}}$ siècle ap. J.-C., pour disparaître ensuite complètement ? Cette intervention des argentarii dans les ventes aux enchères, surtout visible en Italie et qui se répand aussi dans la partie occidentale de l'Empire (mais sans jamais pénétrer dans sa partie orientale, de tradition culturelle grecque), s'explique plus facilement par des raisons économiques et sociales extérieures aux métiers bancaires que par une évolution propre de ces métiers et des opérations qu'ils effectuaient.

En Italie péninsulaire, des enchères ont lieu même dans des centres urbains peu importants, pour deux raisons. Primo, elles jouent un rôle dans la gestion des patrimoines des aristocrates et du reste des notables, qui, collectivement, ont connu un fort enrichissement et à qui il arrive fréquemment de vendre tel ou tel élément de leur patrimoine (en particulier des esclaves), par exemple à la suite d'héritages. Secundo, elles stimulent l'activité suscitée par l'approvisionnement de Rome. Si en effet la cité, à partir de la fin du II siècle av. J.-C., puis l'Empereur, interviennent de façon continue pour fournir à la population de Rome, d'abord du blé à prix réduit, ensuite du blé gratuit, la production et le commerce des autres denrées agricoles demeurent à ces époques presque totalement libres. Rien dans les techniques de changeurs-banquiers ne les prédisposaient particulièrement à s'intéresser aux ventes aux enchères; les trapézites grecs, dont ils avaient hérité les opérations, ne s'en mêlèrent jamais. Ce sont la croissance de la population de Rome et les transformations sociales de la classe des propriétaires fonciers qui sont à l'origine de ce « crédit d'enchères » accordé par les argentarii.

Disons maintenant quelques mots d'une autre évolution, non moins intéressante à mon avis. Probablement au II ${ }^{\mathrm{e}}$ siècle av. J.-C., apparaît dans la cité de Rome ce qu'on appelait en latin le receptum argentarii. Le receptum, qui n'a pas son équivalent dans la pratique bancaire grecque, est un engagement du banquier, auprès d'un tiers, à payer la dette déjà constituée de son client. Il faut supposer que le banquier demandait habituellement un ordre écrit à son client ; mais, en principe, un tel ordre n'était pas nécessaire à la création d'un receptum (le client n'était pas partie prenante au receptum). A quoi servaient concrètement de tels engagements? Dans certains cas, ils permettaient au client de s'acquitter d'un paiement à une date déterminée, sans s'en occuper lui-même, et même éventuellement en son absence. Dans d'autres cas, c'était probablement une manière, pour le banquier, de se porter garant de son client. Car le receptum n'éteint pas l'obligation; le banquier promet de payer à la place du client, mais ce dernier n'en demeure pas moins débiteur tant que le paiement n'a pas eu lieu. Dans d'autres cas encore, le receptum permet au banquier d'ouvrir un crédit à son client, à condition que le client sache déjà à quoi il destine l'argent de ce crédit, et qu'il ait déjà contracté une obligation.

Vers la même époque ou un peu plus tard, au $1^{\mathrm{er}}$ siècle av. J.-C., la notion de compte de dépôts, qui existait déjà dans le monde grec classique, est très renforcée à Rome, surtout dans la pensée juridique. Une séparation très nette est établie entre ceux qui ont le droit d'ouvrir des comptes et ceux qui n'en ont pas le droit. Au sein des activités des argentarii, les opérations qui relèvent du compte sont clairement distinguées de celles qui n'en relèvent pas. Une opération qui est extérieure au compte (extra rationem) est aussi étrangère au métier de banquier, à la banque (extra mensam). En tant qu'argentarius, l'homme de métier doit produire ses registres en justice à la demande de son client, si ce dernier est engagé dans un procès. Mais, à moins d'être lui-même personnellement impliqué dans ce procès, il n'a pas à faire état des opérations extérieures au compte, parce que de telles opérations sortent des limites de son métier de banquier. 
16 Il y a donc là l'ébauche d'un droit professionnel, mais centré sur le compte plutôt qu'autour d'un corps de métier, d'une " corporation» (au sens large du mot). A Athènes au IV siècle av. J.-C., le droit professionnel relatif au commerce et à la banque se définissait au contraire par la nature professionnelle de ceux qu'il visait; on parlait de dikai emporikai ou de dikai trapezitikai, de procédures propres aux négociants ou de procédures propres aux banquiers. La différence est, certes, relative, car ceux qui ont le droit d'ouvrir un compte, ce sont précisément les argentarii, et eux seuls (au moins jusqu'au II ${ }^{\mathrm{e}}$ siècle ap. J.-C.) ; mais elle n'est pas sans importance culturelle et idéologique.

17 A quoi l'attribuer? D'une part à des situations différentes. Ce qui importe avant tout à Athènes, dans le monde des multiples cités-Etats indépendantes, ce sont les rapports avec les professionnels étrangers ; aussi définit-on des groupes relativement faciles à cerner. A Rome, au contraire, le problème des rapports internationaux, à l'époque qui nous intéresse ici, ne se pose plus de la même façon. Mais il y a en outre des explications juridiques. Les $\mathrm{II}^{\mathrm{e}}$ et $\mathrm{I}^{\mathrm{er}}$ siècles av. J.-C. sont des époques de profondes transformations juridiques (songeons à l'édit du préteur et à la procédure formulaire); et, qui plus est, Rome se caratérise désormais par la logique et la vigueur de sa doctrine juridique, qui n'ont pas d'équivalent dans le droit grec classique. La pensée et la mentalité économiques étant très étroitement intégrées à cette élaboration juridique, il ne faut pas s'étonner que les techniques financières en subissent fortement l'influence. Dans le cas du receptum et du compte bancaire, c'est l'inspiration juridique romaine qui conduit à modifier les opérations et techniques financières reçues des Grecs. Mais il ne faut pas oublier que cette élaboration juridique évolue; au $1^{\mathrm{er}}$ siècle av. J.-C., elle est bien différente de ce qu'elle était en 318 av.J.-C., quand les argentarii commencèrent à s'installer dans les boutiques du forum.

18 Le changement que signifie l'apparition du receptum ne s'observe pourtant pas dans l'ensemble des techniques de paiement. Sauf le receptum, tout ce qui existe à Rome en matière de paiements bancaires à des tiers et en matière de virements se rencontre chez Plaute, et, en première approximation, tout ce qui se rencontre chez Plaute était déjà attesté dans l'Athènes classique. Par la suite, les choses n'évoluent pas à cet égard (si les reconnaissances de dettes, quittances et ordres de paiement écrits sont sûrement de plus en plus fréquents, le chèque n'est pas utilisé, et il n'y avait pas de clearing, même entre banques d'une même cité) ; entre le $\mathrm{I}^{\mathrm{er}}$ siècle av. J.-C. et le $\mathrm{II}^{\mathrm{e}}$ siècle ap. J.-C., les allusions aux paiements par l'intermédiaire d'une banque se font rares dans les textes littéraires et juridiques. Pour l'ensemble du service de caisse, il y a stagnation, peut-être même étiolement, dépérissement partiel des techniques mises au point par les Grecs.

19 L'étude des registres professionnels des banquiers ne nous sera malheureusement pas d'une grande aide. Le type de comptabilité que tenaient les Athéniens qui n'étaient pas banquiers est très mal connu et paraît très mal défini. A l'inverse, on sait qu'à Rome, sous la République, tout citoyen aisé tenait le codex accepti et expensi, livre de caisse (en général composé de tablettes de bois garnies de cire ou de laque) sur lequel il notait ses encaissements et ses débours. La disposition de ce livre donne lieu à maintes discussions ; quoique les Romains ne connussent point la numération de position, il se composait probablement de deux pages qui se faisaient face, l'une consacrée aux encaissements ( acceptum) et l'autre aux débours (expensum, depensum). Il était tenu mensuellement à partir de notes prises chaque jour sur une espèce de cahier à tout faire, les adversaria. Au début de l'Empire, il parait tomber en désuétude et, désormais, les particuliers tiennent probablement des comptes séparés, dont certains concernent tel ou tel élément de leur 
patrimoine ou telle ou telle denrée (Caton l'Ancien, déjà, parlait, au $\mathrm{II}^{\mathrm{e}}$ siècle av. J.-C., de ratio frumentaria, comptabilité concernant le blé, et de ratio argentaria, compte des opérations en monnaie), ou telle catégorie d'opérations (le kalendarium est un échéancier de tous les prêts accordés par un particulier).

Quand on passe des registres tenus par n'importe quel citoyen ou résident aux registres professionnels des banquiers, on se trouve devant une situation exactement inversée. Dans l'Athènes classique, la documentation est relativement claire. Les trapézites y tenaient un livre-journal où ils inscrivaient à la suite, chronologiquement, toutes les opérations, sans les classer par comptes, par clients. Certains supposent en outre un grand livre où tout aurait été classé par comptes, mais sans aucune preuve; comme l'a bien montré R. Bogaert, il faut rejeter leur hypothèse. A Rome, au contraire, il est très difficile d'aboutir à des conclusions satisfaisantes. Sous l'Empire, le plus probable est que les argentarii, puis les nummularii aient tenu, comme ceux de l'Athènes classique, un livrejournal, qui était disposé selon l'ordre chronologique, et sans séparation des divers comptes, le livre des rationes.

21 Mais aux derniers siècles de la République ? D'après les textes de Plaute et de Cicéron, il est impossible de décider si, à leurs époques, le banquier de métier tient le codex accepti et expensi au même titre que les autres citoyens ou s'il a un registre professionnel qui lui soit propre. Le problème se complique du fait que, dans le cadre des ventes aux enchères, il tient un registre spécifique, mais qui n'est pas celui des comptes de ses clients; ce sont les tabulae auctionariae ou auctionales, où sont mentionnées toutes les ventes effectuées, avec la nature de l'objet, le nom du vendeur et celui de l'acheteur, le prix intervenu. On ignore donc si les banquiers romains ont emprunté à leurs confrères grecs la nature et la disposition de leur registre professionnel et sont par la suite restés fidèles à cet héritage, ou s'ils se sont conformés d'abord à l'usage romain du codex accepti et expensi, avant de revenir, sous le Haut Empire, à un livre de comptes analogue à celui des banquiers grecs. La première hypothèse paraît, certes, plus simple et vraisemblable que la seconde. Quoiqu'il en soit, l'évolution, en matière de registres, n'est pas comparable, en tout état de cause, à celle que nous avons observée pour le crédit d'enchères, pour le receptum et le compte de dépôts.

En matière de registres, on n'observe nullement la succession d'une première phase, très voisine du modèle grec, et d'une seconde phase, qui le serait moins.

Nous nous trouvons à l'intérieur d'une seule et même civilisation, de sociétés qui évoluent à la fois parallèlement et en étroite symbiose, et qui partagent les mêmes caractéristiques principales. Il est d'autant plus intéressant de comparer l'évolution de leurs techniques financières (ou commerciales : des remarques comparables à celles que j'ai faites pour la banque pourraient s'appliquer au commerce maritime, par exemple).

F. De Martino écrit que Rome a repris au monde grec tous ses modèles, mais en les romanisant et en les revêtant de formes romaines. C'est vrai sur certains points, par exemple quand le droit est en jeu. Mais ce n'est pas vrai pour tout. On constate l'existence de transformations relativement brusques, qui éloignent les techniques romaines, d'abord semblables à celles de la Grèce, de leur modèle originel. Souvent, ces transformations n'ont pas pour effet de "romaniser" la technique grecque, elles marquent une invention, une voie nouvelle qui n'était observable précédemment ni à Athènes ni à Rome - ni ailleurs, pour autant qu'on puisse le savoir. Pensons au cas du crédit dans les enchères ou à celui du receptum. Sur d'autres points, il y a continuité 
durable, fidélité au modèle; c'est en gros le cas pour l'essai des monnaies, ce pourrait l'être aussi pour les registres.

Les mêmes banques athéniennes ont à la fois servi de modèles à celles de Rome et à celles de l'Egypte des Ptolémées. Il y avait pourtant de fortes différences entre les techniques des unes et celles des autres. En Egypte, l'usage du chèque (non endossable) est maintenant assez bien attesté, alors qu'il paraît inconnu à Rome; le crédit d'enchères n'existe pas en Egypte; les rapports entre les banquiers et l'Etat ne sont pas identiques non plus. Mais deux traits sont perceptibles aussi dans l'évolution des techniques bancaires en Egypte: d'une part, une certaine "égyptianisation» des techniques grecques, une certaine adaptation aux conditions économiques, sociales, politiques $\mathrm{du}$ pays; d'autre part, une évolution autonome, qui aboutit à des innovations (bonnes ou mauvaises, peu importe) par rapport à ce qu'on connaissait auparavant, tant en Grèce qu'en Egypte.

Autre conclusion : comment expliquer le jeu de ces évolutions, de ces innovations ou de ces permanences? Les causes sont, sauf exception, extérieures à la logique spécifique des opérations et des techniques. Ce sont des impératifs nouveaux, d'ordre économique, social, politique, administratif, culturel, qui font évoluer les techniques bancaires. L'apparition du crédit d'enchères, qui joue par la suite un rôle très important dans l'activité des banquiers romains, n'est en aucune manière le développement naturel de leurs opérations précédentes. Il s'agit d'une grande nouveauté, d'un cas unique dans l'histoire, et cette nouveauté est une réponse parmi d'autres aux problèmes posés par le ravitaillement de la ville de Rome; elle marque en outre une adaptation aux manières nouvelles qu'ont les élites romaines de gérer leur patrimoine et à l'évolution de la composition de ce patrimoine. La stagnation du service de caisse n'était pas inéluctable; elle résulte de l'évolution du rôle social et économique des banquiers romains, dont les clientèles paraissent de moins en moins élevées dans la hiérarchie sociale (au II ${ }^{\mathrm{e}}$ siècle av. J.-C., le sénateur Scipion Emilien avait ouvert un compte en banque; au $1^{\text {er }}$ siècle av. J.-C. et sous le Haut Empire, nous ne connaissons plus un sénateur qui soit titulaire d'un tel compte).

Les banquiers n'en sont pas moins utiles pour cela, il est même probable que leurs rapports avec les milieux du commerce s'en trouvent renforcés. Mais, en conséquence de cette perte de rang social, ils n'ont plus besoin d'innover en matière de transferts de fonds, en matière de paiements à des tiers sur ordre du client, de chèques, de virements, etc. Entre l'époque de Périclès et le $\mathrm{III}^{\mathrm{e}}$ siècle av. J.-C., les banques grecques ont réuni peu à peu tout un patrimoine technique, dont une partie sortira, plus tard, appauvrie par l'évolution économique et sociale du monde romain. Ainsi, le service de caisse, fortement attesté jusqu'à l'époque de Plaute, paraît perdre de l'importance par la suite. Mais l'époque romaine élabore d'autres techniques bancaires et, par ailleurs, il ne faut oublier ni l'activité financière des aristocrates (qui n'étaient pas des professionnels) ni celle des sociétés de publicains. Entre le $\mathrm{V}^{\mathrm{e}}$ siècle av. J.-C. et le $\mathrm{II}^{\mathrm{e}}$ siècle ap. J.-C., tous les secteurs de la vie financière grecque et romaine sont donc bien loin de connaitre une seule et même évolution technique.

\section{Bibliographie}



Paris, C.N.R.S., 1988 (au sujet des pierres de touche trouvées sur des épaves, voir pp. 92 et 148-149). Economy, P. Garnsey, K. Hopkins et C. R. Whittaker (éd.), Londres, Chatto et Windus, 1983, pp. 36-52.

Cl. PREAUX, « De la Grèce classique à l'Egypte hellénistique, la banque-témoin », Chronique

J. ANDREAU, Vie financière dans le monde romain, Les Métiers de manieurs d'argent (IV siècle av. J.-C. - III siècle ap. J.-C.), Rome, École Française de Rome, 1987.

R. BOGAERT, Banques et banquiers dans les cités grecques, Leyde, Sijthoff, 1968.

R. BOGAERT, «L'essai des monnaies dans l'Antiquité », Revue belge de Numismatique, 122, 1967, pp. 5-34.

R. BOGAERT, « Note sur l'emploi du chèque dans l'Egypte ptolémaïque », Chronique d'Egypte, 58, 1983, pp. 212-221.

F. de MARTINO, Storia economica di Roma antica, Florence, Nuova Italia, 1980.

L. GERNET, Droit et société dans la Grèce ancienne. Paris, Sirey, 1964.

A. HESNARD ET ALII, L'épave romaine Grand Ribaud D (Hyères, Var) (=Archaeonautica, 8), M. LOMBARDO, «Marchands, économie et techniques d'écriture », dans Les Savoirs de l'écriture. En Grèce ancienne, M. Detienne (éd.), Lille, P.U.L., 1988, pp. 159-187.

P. MILLETT, «Maritime loans and the structure of credit» dans Trade in the Ancient d'Egypte, 33, 1958, pp. 243-255. d'Egpte, 33, 1958, pp. 243-255. 